\title{
Optically Trapped Bacteria Pairs Reveal Discrete Motile Response to Control Aggregation upon Cell-Cell Approach
}

\author{
Maria Dienerowitz • Laura V. Cowan • \\ Graham M. Gibson · Rebecca Hay • \\ Miles J. Padgett $\cdot$ Vernon R. Phoenix
}

Received: 10 March 2014 / Accepted: 5 May 2014/Published online: 26 June 2014

(C) The Author(s) 2014. This article is published with open access at Springerlink.com

\begin{abstract}
Aggregation of bacteria plays a key role in the formation of many biofilms. The critical first step is cellcell approach, and yet the ability of bacteria to control the likelihood of aggregation during this primary phase is unknown. Here, we use optical tweezers to measure the force between isolated Bacillus subtilis cells during approach. As we move the bacteria towards each other, cell motility (bacterial swimming) initiates the generation of repulsive forces at bacterial separations of $\sim 3 \mu \mathrm{m}$. Moreover, the motile response displays spatial sensitivity with greater cell-cell repulsion evident as inter-bacterial distances decrease. To examine the environmental influence on the inter-bacterial forces, we perform the experiment with bacteria suspended in Tryptic Soy Broth, $\mathrm{NaCl}$ solution and deionised water. Our experiments demonstrate that repulsive forces are strongest in systems that inhibit biofilm formation (Tryptic Soy Broth), while attractive forces are weak and rare, even in systems where biofilms develop ( $\mathrm{NaCl}$ solution). These results reveal that bacteria are able to control the likelihood of aggregation during the approach phase through a discretely modulated motile response. Clearly, the force-generating motility we observe during approach promotes biofilm prevention, rather than biofilm formation.
\end{abstract}

M. Dienerowitz $(\bowtie) \cdot$ L. V. Cowan · G. M. Gibson .

R. Hay · M. J. Padgett

SUPA, School of Physics and Astronomy, University of

Glasgow, Glasgow G12 8QQ, UK

e-mail: maria.dienerowitz@glasgow.ac.uk

V. R. Phoenix

School of Geographical and Earth Sciences, University of

Glasgow, Glasgow G12 8QQ, UK

\section{Introduction}

Biofilms are exploited in a wide range of biotechnologies, including wastewater treatment, biofuel production and the generation of electricity in microbial fuel cells $[16,21]$. Conversely, however, they generate billions of dollars in losses each year through machinery damage, loss of processing and manufacturing efficiency, product contamination and medical infections [9, 12, 17, 22]. As a result, understanding the mechanisms of biofilm formation have become key to both the design of biofilm for optimal biotechnological use and the development of biofilm inhibitors preventing medical infections or equipment damage. Aggregation of planktonic cells, either to each other or to a biofilm, plays a key role in biofilm formation $[1,15,18,23]$. Successful aggregation is driven on close contact between bacterial cells by physical forces such as attractive van der Waals and biological mechanisms, for example the bridging of protein adhesins and saccharide receptors between opposing cell walls $[10,15]$. However, before these mechanisms can induce aggregation, bacteria must first approach each other either via swimming motility or through Brownian motion. While approach of planktonic cells is the critical first step in aggregation, we know nothing of how individual bacteria control this process.

Here, we measured the force generated during approach of bacterial nearest neighbours using Bacillus subtilis cells trapped with optical tweezers (Fig. 1a). Although an optical trap restricts the bacterium's position in space, its non-invasive nature leaves the bacterium's natural motion unperturbed enabling its motile response to be analysed [2, 14, 19]. To measure the forces between two individual Bacillus subtilis cells, we held one cell in a fixed position (static trap) and tracked its motion, while we positioned a second cell (moving 
a

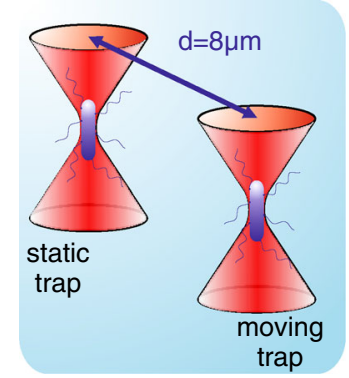

b

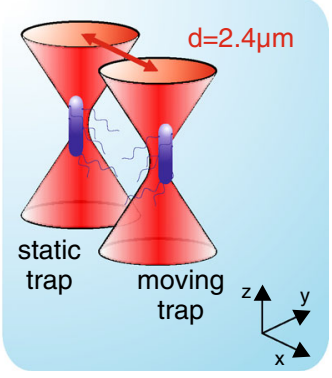

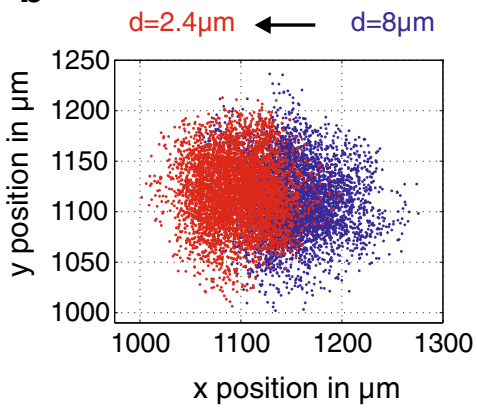

C

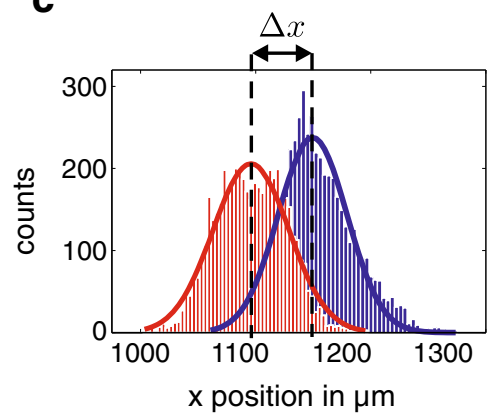

Fig. 1 Optical force measurements between individual Bacillus subtilis bacteria. a Two optical traps hold a bacterium each at a distance varying between 2 and $8 \mu \mathrm{m}$. b For every separation distance $d$ of each bacterial pair, one measurement records 50,000 positions of the bacterium in the static trap. $\mathbf{c}$ The force between the two bacteria is determined from the shift of the mean position $\Delta x$ of the bacterium in the static trap relative to its starting position at large separation distances $(d=6-8 \mu \mathrm{m})$ where no bacteria interaction is assumed trap) within close vicinity. Recording the mean position of the bacterium in the static trap and monitoring its shift away from the trap centre, $\Delta x$ quantifies the external force induced by the bacterium in the moving trap (Fig. 1b, c). According to Hooke's law $F=-\kappa \Delta x$ (with the trap stiffness $\kappa$ ), we obtain a precise measurement of the force $F$ exerted on the trapped bacterium in the static trap. To stimulate different levels of aggregation, we performed the experiments in three different media: Tryptic Soy broth (TSB), deionized water and 0.1 M $\mathrm{NaCl}$ solution. Deionized water and $0.1 \mathrm{M} \mathrm{NaCl}$ solution were chosen as they provided nutrient limited conditions, which promote biofilm formation in many bacteria, including $B$. subtilis [11,25]. Indeed, we observed numerous aggregates of bacterial cells forming under $0.1 \mathrm{M} \mathrm{NaCl}$ solution, with occasional bacterial aggregates occurring in deionized water and none for cells suspended in TSB.

\section{Materials and Methods}

\section{Holographic Optical Tweezers}

Figure 2 shows a schematic diagram of the inverted optical tweezers setup, and an extensive description is detailed elsewhere [13]. In essence, we split a Ti:Sapphire laser beam $(830 \mathrm{~nm})$ with a spatial light modulator (SLM, Boulder Nonlinear Systems) and focussed it tightly with a $100 \times$ Nikon microscope objective (NA 1.3) into the sample chamber. The SLM controls the two generated optical traps independently, and they are positioned $10 \mu \mathrm{m}$ deep in the sample to avoid any interaction of the bacteria with the glass cover slip. We kept the laser power in each trap very low $(3-5 \mathrm{~mW})$ to avoid damaging the bacteria and to provide as much possible freedom for a motile response. Trapping bacteria at low power in a weak trap results in a low trap stiffness, which in turn enables us to measure very small forces. The bacteria actively swam

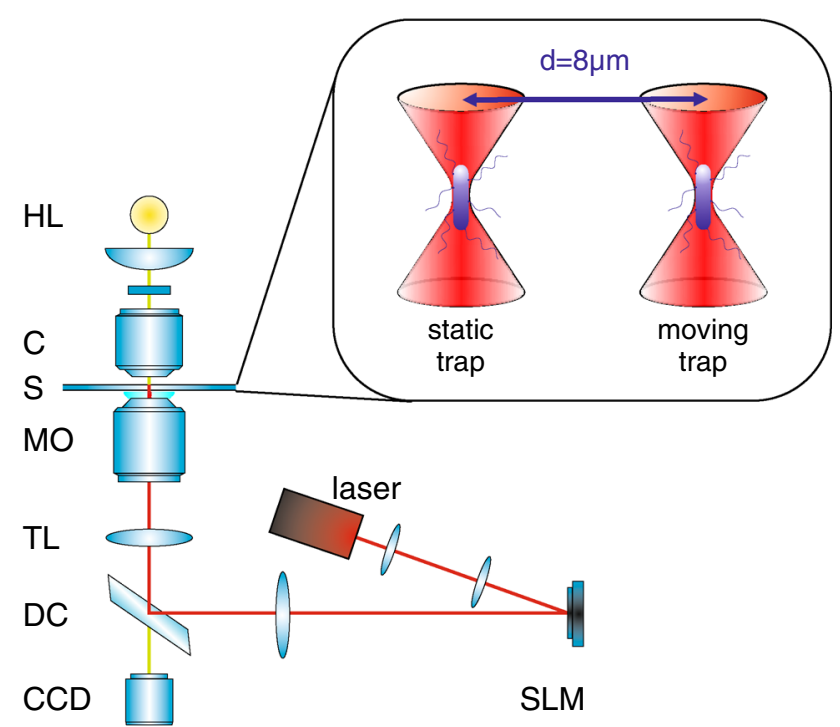

Fig. 2 Schematic of the experimental setup. A Ti:Sapphire laser (M Squared, SolsTiS, $1.5 \mathrm{~W}, 790-850 \mathrm{~nm}$ ) is split into two traps by a spatial light modulator (SLM, Boulder Nonlinear Systems, XY Series, $512 \times 512$ pixels). After passing a dichroic mirror $(D C)$ and the tube lens $(T L)$, the laser enters the microscope objective $(M O$, Nikon CFI Plan Fluor, oil immersion, NA 1.3) and is focussed into the sample chamber (S), which contains the bacteria solution sandwiched between two glass coverslips. A halogen bulb $(H L)$ illuminates the sample through the condenser (C, Zeiss NA 0.9 ), and a $C C D$ camera (Prosilica GE680C) records the images of the bacteria in the traps. A sketch of the trap setup inside the sample cell is displayed in the inset

away from the trap site once we turned off the trapping laser, which indicates good bacterial viability even after longer periods of trapping. We performed all experiments at room temperature $\left(22^{\circ} \mathrm{C}\right)$.

Data Acquisition and Force Measurements

We keep the first trap at the same position at all times (static trap), whereas we move the second trap laterally with respect 
to the first in a step-wise fashion. During position measurement, both traps remain stationary. In order to avoid an overlap of the optical trapping potentials, we did not move the traps closer than $2 \mu \mathrm{m}$. The bacteria's average diameter is $500 \mathrm{~nm}$, which leaves approximately $1 \mu \mathrm{m}$ between cell walls at the closest separation. For each set distance between the static and the moving trap, we take 50,000 position measurements of the bacterium in the static trap along its short axis. We continue by positioning the moving trap closer to the static trap in $100 \mathrm{~nm}$ steps, taking a measurement every $500 \mathrm{~nm}$ and for separation distances below $3 \mu \mathrm{m}$ every $200 \mathrm{~nm}$. Once the bacteria are $2 \mu \mathrm{m}$ apart we separate them again, tracing back the positions of the moving trap and repeat measuring the position of the bacteria in the static trap every 200/500 $\mathrm{nm}$ as on approach. The position measurements at each separation distance take $25 \mathrm{~s}$ to complete. Overall, measuring the positions of one bacterial pair as well as moving the traps close together and separating them again last for $20 \mathrm{~min}$.

We apply Hooke's law to obtain the force from the displacement $\Delta x$ of the mean position of the bacteria in the trap: $F=-\kappa x \Delta$ with the trap stiffness $\kappa=k_{\mathrm{B}} T /\left(\sigma_{\mathrm{x}}\right)^{2}$. Here, $k_{\mathrm{B}}$ is Boltzmann's constant, the temperature is $T=295.15 \mathrm{~K}$ and $\sigma_{\mathrm{x}}$ is the standard deviation of the measured position data. We measure the trap stiffness $\kappa$ for every separation distance and average all the obtained values for $\kappa$ over one bacterial pair. The mean position of a trapped $1 \mu \mathrm{m}$ polystyrene bead in our control experiment varies between $\pm 3 \mathrm{~nm}$, which is within the experimental error limit. We track the trapped bacteria's motion by recording the video images for the particle tracking analysis with a high-speed camera (Prosilica GE680C) at 2,000 Hz. We control all devices as well as the image acquisition and particle tracking with customised LabVIEW software (National Instruments) created by Bowman [6].

\section{Bacteria Sample Preparation}

Bacillus subtilis cultures (ATCC 23,857) were grown in Tryptic Soy Broth at $30{ }^{\circ} \mathrm{C}$ on a rotary shaker at $130 \mathrm{rpm}$ for $\sim 18 \mathrm{~h}$. We centrifuged $0.5 \mathrm{ml}$ of culture for $2 \mathrm{~min}$ at $1,000 \mathrm{rpm}$ and subsequently removed the supernatant media. The remaining bacteria were transferred into new media (either TSB, deionized water or $0.1 \mathrm{M} \mathrm{NaCl}$ solution). Using a plastic pipette, we applied the bacteria in the new media to a single concave microscopic slide and sealed the cover slip to the slide with petroleum jelly.

\section{Results}

The first set of experiments investigated B. subtilis cells suspended in TSB. As we decreased the separation distance $d$ between bacteria, a majority of bacteria in the static trap began to show repulsion (they repelled themselves from their neighbour in the moving trap) at a centre-to-centre distance of approximately $3.5 \mu \mathrm{m}$ (Fig. 3a, c). Intriguingly, as the distance between the bacteria decreased, cells commonly showed an increase in repulsive force, reaching an average maximum of 0.25 $\mathrm{pN}$ at a centre-to-centre separation of $2 \mu \mathrm{m}$ (Fig. 3a, c). Then, as we moved the cells apart, the repulsive force decreased, following a similar trend to that observed during cell-cell approach (Fig. 3b, c). We repeated the experiment with cells suspended in either $0.1 \mathrm{M} \mathrm{NaCl}$ solution or deionized water (Figs. 4a, b, 5). In deionised water, again, a majority of cells displayed an increase in repulsive force as they moved closer, but the maximum average repulsive force $(0.09 \mathrm{pN})$ was considerably weaker than in TSB $(0.25 \mathrm{pN})$. As before, the repulsive force decreased as the optical traps moved the cells apart, closely tracking the trend observed on approach (Figs. 4b, 5a). We measured no significant repulsive force undertaking identical experiments in $0.1 \mathrm{M} \mathrm{NaCl}$ solution (Figs. 4a, b, 5b).

Overall, our results show that the strongest repulsive forces between two individual bacteria occur under nutrient-rich conditions, which did not promote aggregation. Conversely, weaker or absent repulsive forces dominated in systems where aggregation occurred (deionized water or $0.1 \mathrm{M} \mathrm{NaCl}$ solution). Thus, we suggest the strong cell-cell repulsion seen on approach plays a role in preventing aggregation in the nutrient-rich TSB, while the weaker or absent repulsive forces facilitate aggregation in the nutrient-starved systems. Notably, significant attractive forces were rare and did not show systematic trends. Evidently, attractive forces are not required on approach to promote aggregation. Instead, when forces are generated on approach, they are repulsive in nature and dedicated to prevent aggregation.

We argue that the repulsive forces observed must result from cell motility (bacterial swimming). We can categorically exclude electrostatic repulsion, because these forces only extend tens of nanometres from the cell surface [17]; thus, cannot explain the repulsion we saw at separations of microns. While a majority of cells in TSB and deionized water displayed motile repulsion, there was significant variability in the motile force generated by different bacterial pairs. This was most notable in TSB where forces ranging from zero to $0.62 \mathrm{pN}$ at the closest separations. Clearly, even in TSB, some cells displayed significant repulsion while others displayed none. However, B. subtilis cells from a single culture are known to display intracellular variability in the extent of motility [5]; thus, we argue that significant variations in the extent of the repulsive force must be expected. 
a

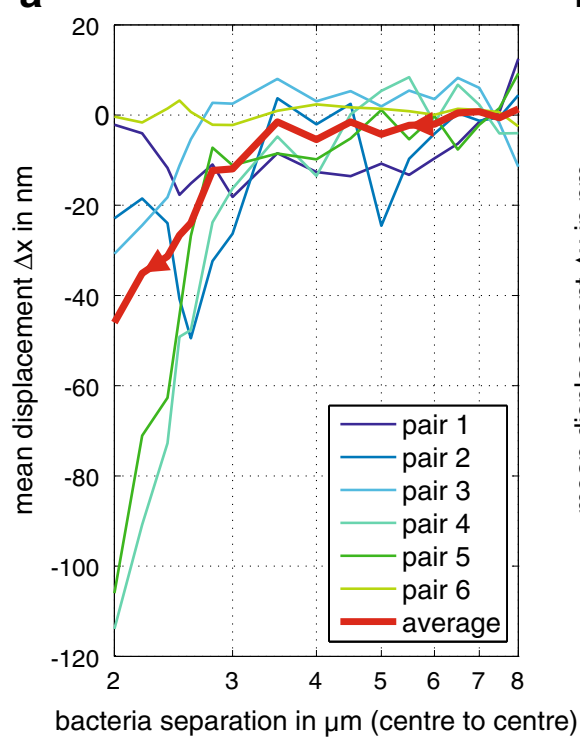

b

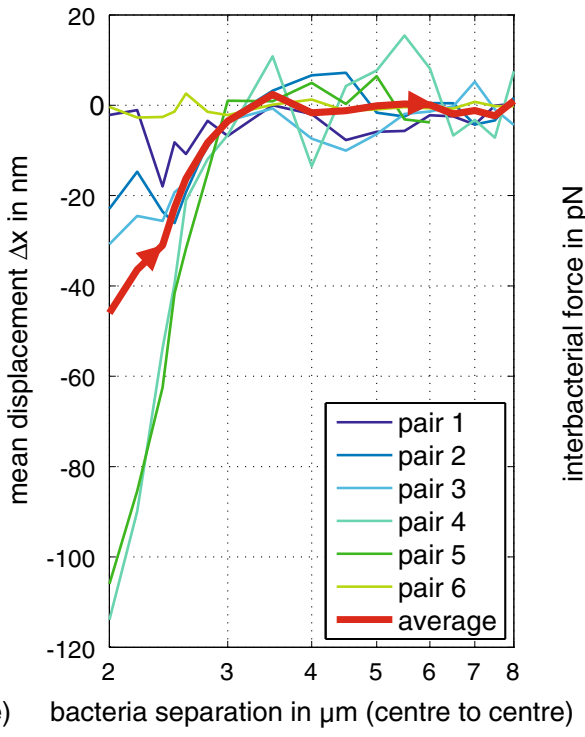

C

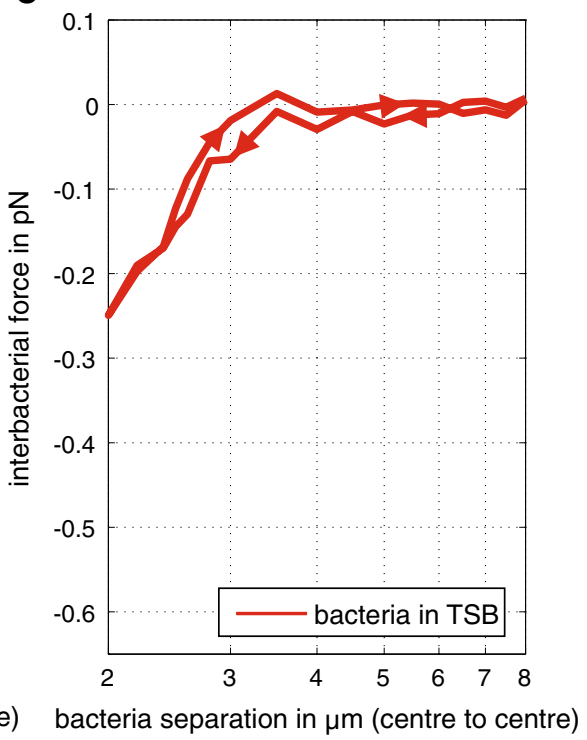

Fig. 3 Mean displacements and inter-bacterial forces between bacterial pairs in Tryptic Soy Broth (TSB). a The mean displacement $\Delta x$ of the bacterium in the static trap from the trap centre increases on approach of the second bacterium in the moving trap. Results are shown for six separate bacterial pairs and their combined average.

As a control, we repeated the experiments in nutrientrich TSB media with deactivated (autoclaved) bacteria, which were killed to prevent motility. We observed no repulsion (Fig. 4c, d), supporting the hypothesis that motility appears to be responsible for repulsion observed for functioning bacteria. Conspicuously, a small attractive force of $0.04 \mathrm{pN}$ was observed at the closest separation. However, our control experiments with $1 \mu \mathrm{m}$ polystyrene beads showed that this small attractive force was within the force variability displayed by the beads (Fig. 4c, d). As a comparison, the repulsive forces we measured for functioning bacteria in TSB and deionized water exceed at least twice the magnitude of the force variability displayed by polystyrene beads and autoclaved bacteria.

\section{Discussion}

Motility is known to both aid and inhibit biofilm formation. For example, examination of dual species biofilms containing Pseudomonas Aeruginosa and Agrobacterium tumefaciens reveals that A. tumefaciens cells use motility as an escape mechanism to prevent incorporation into the biofilm [3]. A. tumefaciens cells, however, were already a part of the biofilm. Our work here reveals that motile escape mechanisms can exist prior to aggregation, thus inhibiting the earliest steps of biofilm formation. Equally, upregulation of flagella synthesis and motility genes has been shown to stimulate b The mean displacement $\Delta x$ of the bacterium in the static trap from the trap centre on retreat of the second bacterium closely follows the trend on approach. c Summary of average inter-bacterial forces calculated from $\Delta x$ on approach and retreat. All measurements are corrected for drift

biofilm formation; the upregulation was directed by quorum sensing [4]. Quorum sensing enables bacteria to detect the relative density of their surrounding bacterial population via the production of autoinducer molecules. As these accumulate within the cell, upregulation of specific genes occurs above certain concentration thresholds, inducing a wide variety of responses, including biofilm formation [24, 26]. Most importantly, quorum sensing is a community-wide response-a response to the bulk density of the surrounding population. It has not yet been shown to facilitate communication between two individual bacteria, nor their spatial awareness of each other.

In our study, the close relationship between separation distance and force suggests a spatial sensitivity between bacterial pairs and a form of communication between the two cells. Our experiments, however, do not reveal the mechanism by which communication occurs. One may speculate that the bacteria are sensing a concentration gradient of a compound excreted by the organism, and thus the motile repulsion is a chemotactic response. Indeed, the ability to influence motility through sensing concentration gradients of excreted compounds has been shown for Escherichia coli $[7,20]$, opening the possibility that an analogous mechanism may be utilised by $B$. subtilis to prevent aggregation. Alternatively, inter-bacterial sensing may result from contact of the flagella of the nearest neighbour. Indeed, B. subtilus cells can display flagella over $3 \mu \mathrm{m}$ long [8], and therefore flagella contact is possible at the distances where we observed cell- 

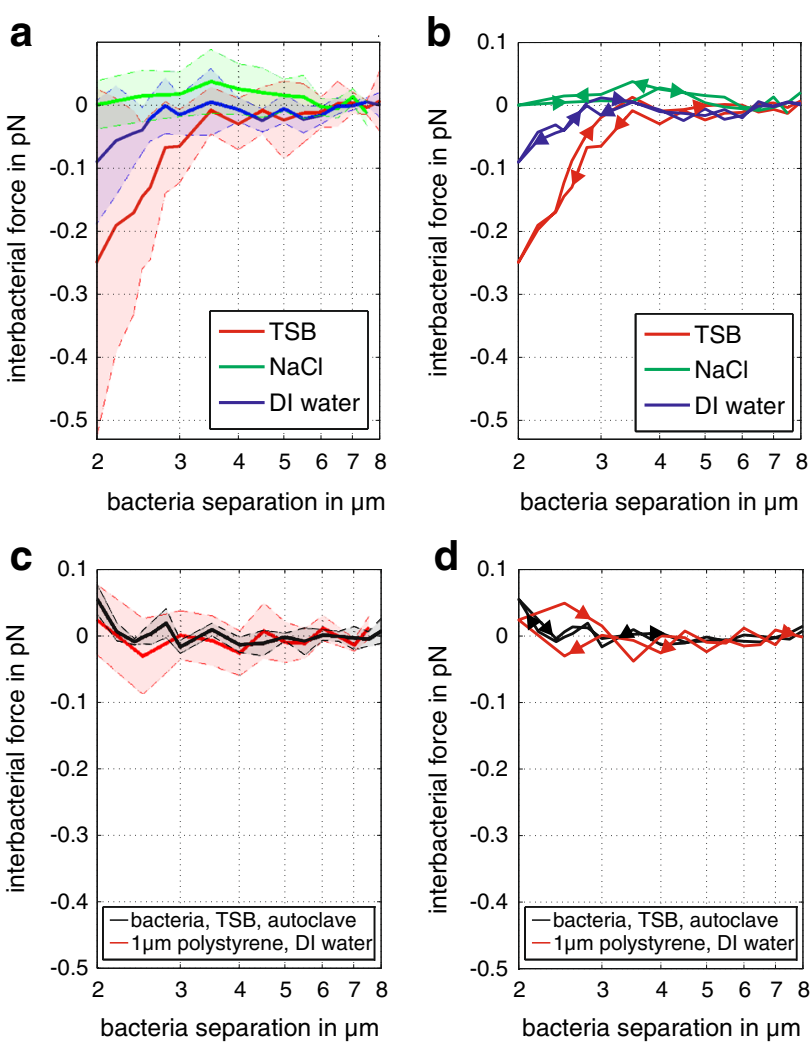

Fig. 4 Environmental influence on inter-bacterial forces. a The repulsive force (each force line represents the average of six bacterial pairs) on approach is strongest for bacteria suspended in TSB, decreases for nutrient-deprived deionized water and disappears for bacteria in $\mathrm{NaCl}$ solution. The shaded areas represent the standard deviation of the force at each separation distance for the six bacterial pairs. b Averaged force lines follow the same trend on approach and retreat. c-d Average force and standard deviation on approach and retreat for autoclaved bacteria in TSB and $1 \mu \mathrm{m}$ polystyrene beads in deionized water show no repulsion, indicating that we observe a biological response in living samples

cell repulsion $(<3 \mu \mathrm{m})$. However, it is unlikely that the physical presence of flagella alone is causing repulsion simply by behaving as a spring mechanism. If this was the case, we would expect to see equal repulsion in all three liquid media. Whatever mechanism is utilised to sense interbacterial distances, it is evident that the bacteria are able to adjust their motile response to control the likelihood of aggregation. Importantly, these are individual responses of nearest neighbours, which act in isolation from the rest of the community. Yet overall, the combined response of individuals leads to the global phenomenon of either biofilm formation or prevention.

Acknowledgments The authors thank EPSRC EP/H007636/1 and the University of Glasgow for financial support. M.J.P. thanks the Royal Society.

Open Access This article is distributed under the terms of the Creative Commons Attribution License which permits any use,
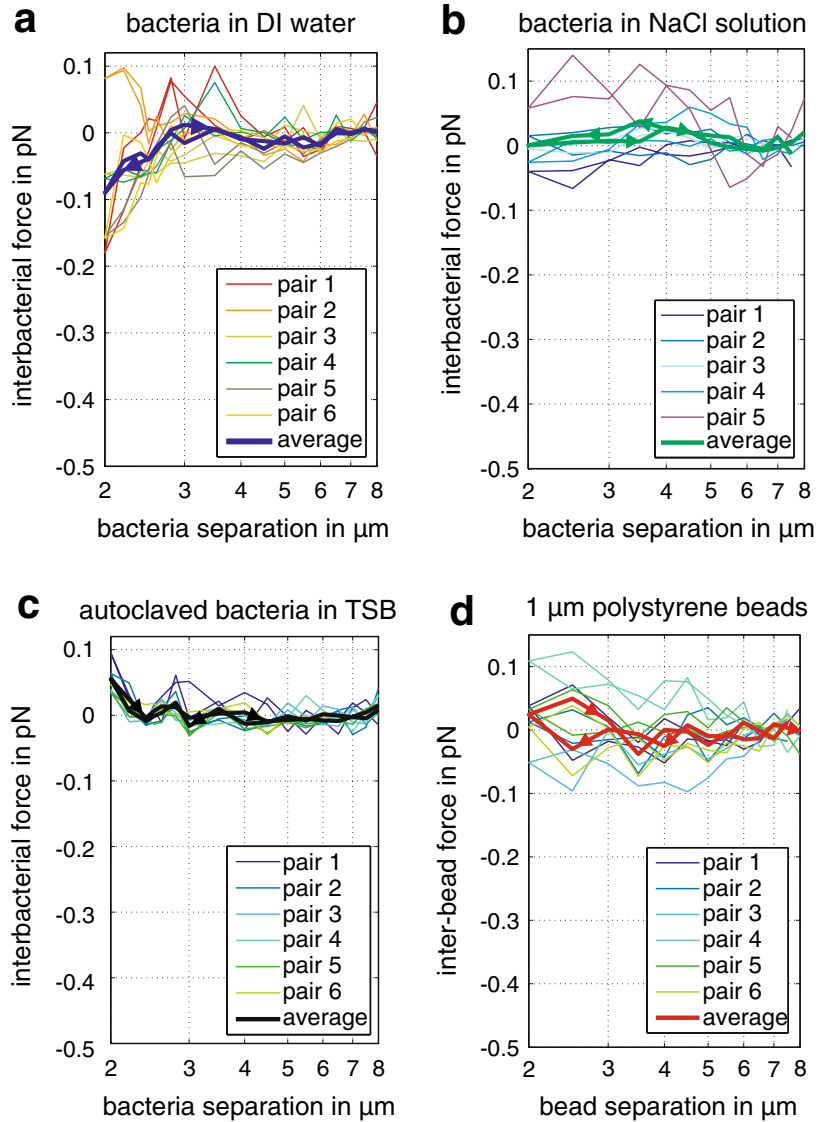

Fig. 5 Environmental influence on inter-bacterial forces. The graphs display the complete datasets for the average force lines displayed in Fig. 4. Force on approach and retreat for bacteria suspended in a deionized water, $\mathbf{b} \mathrm{NaCl}$ solution, $\mathbf{c}$ autoclaved bacteria in TSB and d $1 \mu \mathrm{m}$ polystyrene beads. Each bold line represents the force average of six bacterial pairs or six polystyrene bead pairs. Only the interaction of bacteria in deionized water result in a repulsive force for trap separations below $3 \mu \mathrm{m}$. All other experiments displayed no significant change in force for decreasing the separation distance of the traps. All measurements are corrected for drift

distribution, and reproduction in any medium, provided the original author(s) and the source are credited.

\section{References}

1. Al-Bakri AG, Gilbert P, Allison DG (2004) Immigration and emigration of Burkholderia cepacia and Pseudomonas aeruginosa between and within mixed biofilm communities. J Appl Microbiol 96:455-463

2. Altindal T, Chattopadhyay S, Wu X (2011) Bacterial chemotaxis in an optical trap. PLoS ONE 6:e18231

3. An D, Danhorn T, Fuqua C, Parsek MR (2006) Quorum sensing and motility mediate interactions between Pseudomonas aeruginosa and Agrobacterium tumefaciens in biofilm cocultures. PNAS 103:3828-3833

4. Barrios AFG, Zuo R, Hashimoto Y, Yang L, Bentley WE, Wood TK (2006) Autoinducer 2 controls biofilm formation in Escherichia coli through a novel motility quorum-sensing regulator (MqsR, B3022). J Bacteriol 188:305-316 
5. Blair KM, Turner L, Winkelman JT, Berg HC, Kearns DB (2008) A molecular clutch disables flagella in the Bacillus subtilis biofilm. Science 320:1636-1638

6. Bowman R (2012) Optical tweezers Software, www.gla.ac.uk/ schools/physics/research/groups/optics/research/opticaltweezers/ software

7. Budrene EO, Berg HC (1991) Complex patterns formed by motile cells of Escherichia coli. Nature 349:630-633

8. Cisneros LH, Cortez R, Dombrowski C, Goldstein RE, Kessler JO (2007) Fluid dynamics of self-propelled microorganisms, from individuals to concentrated populations. Exp Fluids 43:737-753

9. Coraca-Huber DC, Fille M, Hausdorfer J, Pfaller K, Nogler M (2012) Staphylococcus aureus biofilm formation and antibiotic susceptibility tests on polystyrene and metal surfaces. J Appl Microbiol 112:1235-1243

10. Das T, Krom BP, van der Mei HC, Busscher HJ, Sharma PK (2011) DNA-mediated bacterial aggregation is dictated by acidbase interactions. Soft Matter 7:2927-2935

11. Elhariry HM (2008) Biofilm formation by endospore-forming bacilli on plastic surface under some food-related and environmental stress conditions. Global J Biotechnol Biochem 3:69-78

12. Flemming HC (2011) Microbial Biofouling: unsolved problems, unsufficient approaches and possible solutions. Biofilm Highlights 5:81-109

13. Gibson GM, Leach J, Keen S, Wright AJ, Padgett MJ (2008) Measuring the accuracy of particle position and force in optical tweezers using high-speed video microscopy. Opt Express 16:14561-14570

14. Kemper B, Barroso A, Woerdemann M, Dewenter L, Vollmer A, Schubert R, Mellmann A, von Bally G, Denz c (2013) Towards $3 \mathrm{D}$ modelling and imaging of infection scenarios at the single cell level using holographic optical tweezers and digital holographic microscopy. J Biophotonics 6:260-266

15. Kolenbrander PE, Palmer RJ Jr, Periasamy S, Jakubovics NS (2010) Oral multispecies biofilm development and the key role of cell-cell distance. Nat Rev Microbiol 8:471-480
16. Logan BE, Hamelers B, Rozendal R, Schröder U, Keller J, Freguia S, Aelterman P, Verstraete W, Rabaey K (2006) Microbial fuel cells: methodology and technology. Environ Sci Technol 40:5181-5192

17. Lynch AS, Robertson GT (2008) Bacterial and fungal biofilm infections. Annu Rev Med 59:415-428

18. Malik A, Sakamoto M, Hanazaki S, Osawa M, Suzuki T, Tochigi M, Kakii K (2003) Coaggregation among nonflocculating bacteria isolated from activated sludge. Appl Environ Microbiol 69:6056-6063

19. Min TL, Mears PJ, Chubiz LM, Rao CV, Golding I, Chemla YR (2009) High-resolution, long-term characterization of bacterial motility using optical tweezers. Nat Methods 6:831-U871

20. Mittal N, Budrene EO, Brenner MP, van Oudenaarden A (2003) Motility of Escherichia coli cells in clusters formed by chemotactic aggregation. PNAS 100:13259-13263

21. Qureshi N, Annous BA, Ezeji TC, Karcher P, Maddox IS (2005) Biofilm reactors for industrial bioconversion processes: employing potential of enhanced reaction rates. Microb Cell Fact 4:24

22. Ren D, Sims JJ, Wood TK (2002) Inhibition of biofilm formation and swarming of Bacillus subtilis by (5Z)-4-bromo-5-(bromomethylene)- 3-butyl-2(5H)-furanone. Lett Appl Microbiol 34:293-299

23. Rickard AH, Gilbert P, High NJ, Kolenbrander PE, Handley PS (2003) Bacterial coaggregation: an integral process in the development of multi-species biofilms. Trends Microbiol 11:94-100

24. Smith RS, Iglewski BH (2003) P. aeruginosa quorum-sensing systems and virulence. Curr Opin Microbiol 6:56-60

25. Stanley NR, Britton RA, Grossman AD, Lazazzera BA (2003) Identification of catabolite repression as a physiological regulator of biofilm formation by Bacillus subtilis by Use of DNA Microarrays. J Bacteriol 185:1951-1957

26. Waters CM, Bassler BL (2005) Quorum sensing: cell-to-cell communication in bacteria. Annu Rev Cell Dev Bi 21:319-346 